\title{
MCBP Regimen
}

National Cancer Institute

\section{Source}

National Cancer Institute. MCBP Regimen. NCI Thesaurus. Code C10028.

A chemotherapy regimen consisting of melphalan, cyclophosphamide, carmustine, and prednisone that may be used in the treatment of plasma cell myeloma. 